\title{
Paraovarian cystic endosalpingiosis in association with tamoxifen therapy
}

\author{
W G McCluggage, P E Weir
}

Department of Pathology, Royal Group of Hospitals Trust, Grosvenor Road, Belfast BT12 6BL, Northern Ireland, UK

W G McCluggage

Department of Obstetrics and Gynaecology, Mater Infirmorum Hospital, Belfast

P E Weir

Correspondence to: Dr McCluggage

Accepted for publication 11 August 1999

\begin{abstract}
This report describes a case of macroscopically visible cystic endosalpingiosis involving the paraovarian region in a woman who had been taking tamoxifen for breast cancer. A $2.5 \mathrm{~cm}$ multicystic lesion was seen on the external surface of the right ovary and histological examination showed a mass of dilated glands lined by ciliated tubal-type epithelium and set in a fibrovascular stroma. Cystic endosalpingiosis resulting in a tumour-like mass is a rarely described entity which is probably not well recognised by histopathologists. Although unlikely to be mistaken for malignancy, the lesion may result in diagnostic confusion. The role of tamoxifen in the development of the lesion in the present case is not clear but the oestrogenic effects of this drug may have contributed to its formation. (F Clin Pathol 2000;53:161-162)
\end{abstract}

Keywords: ovary; paraovarian region; endosalpingiosis, tamoxifen

Endosalpingiosis is a benign condition characterised by the presence of glands lined by ciliated tubal-type epithelium. The condition usually affects women and typically involves the pelvic and abdominal peritoneum. ${ }^{1}$ The glandular epithelium is thought to be derived from peritoneal cells as part of the so called secondary müllerian system and is one of the triad of non-neoplastic secondary müllerian lesions, the others being endometriosis and endocervicosis.

Endosalpingiosis is almost always an incidental finding on microscopic examination and rarely presents as a grossly visible mass. Recently, Clement and Young drew attention to this lesion in describing four cases of florid cystic endosalpingiosis associated with a tumour-like mass. ${ }^{2}$ Our aim in this report is to

ingiosis which resulted in a grossly visible paraovarian mass. Histopathologists may not be aware of this entity and diagnostic confusion may arise. The patient in the present case had been taking tamoxifen for breast cancer and we speculate on the possible role of this drug in the development of cystic endosalpingiosis.

\section{Case report}

A 48 year old woman underwent total mastectomy for breast carcinoma, following which she was started on tamoxifen, $20 \mathrm{mg}$ daily. Approximately three years later, during investigations for urinary symptoms, she was found to have a large polyp which protruded through the external cervical os. This was removed. Over the next two years she suffered from persistent intermittent vaginal bleeding and two further endometrial polyps were removed. Histology of all the lesions showed benign endometrial polyps, similar to those described below. Her tamoxifen treatment was discontinued after five years of continuous therapy.

Because of the development of multiple endometrial polyps, a total abdominal hysterectomy and bilateral salpingo-oophorectomy was performed.

\section{Pathological findings}

The surgical specimen consisted of a uterus without cervix and attached right and left ovaries and fallopian tubes, together weighing 158 g. Sectioning the uterus revealed two endometrial polyps, the larger measuring $2 \mathrm{~cm}$ in maximum dimension. Several intramural fibroids were also present, the largest measuring $3 \mathrm{~cm}$ maximum. Both fallopian tubes measured $4 \mathrm{~cm}$ in length and were grossly unremarkable. Both ovaries measured $5 \mathrm{~cm}$ maximum and each contained a $2 \mathrm{~cm}$ cyst. Related to the superior aspect of the capsular surface of the right ovary, but clearly separate from the ovary, a 2.5 $\mathrm{cm}$ mass was present which on sectioning contained multiple small cysts.
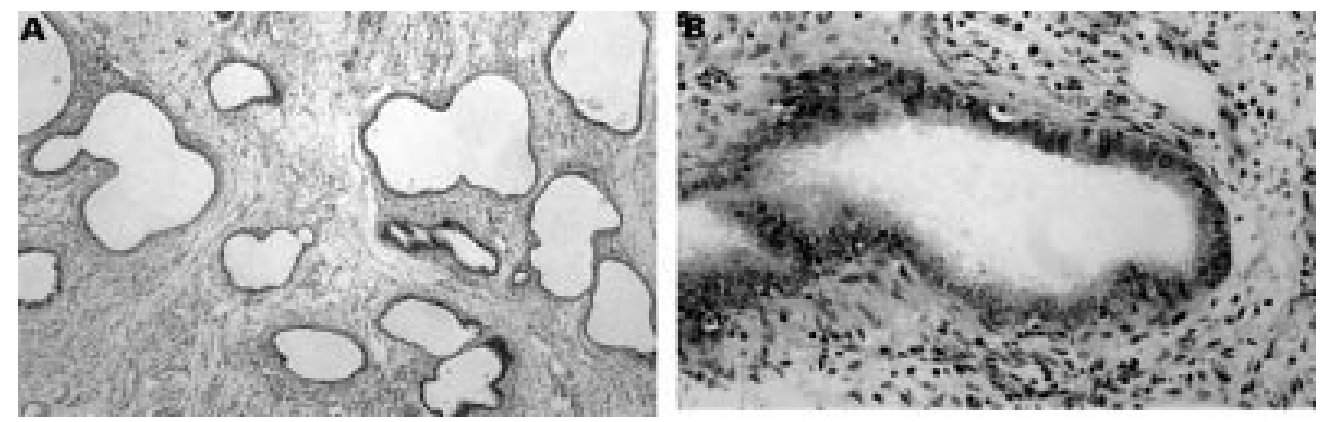

Figure 1 (A) Multiple cyst-like spaces lined by a single layer of epithelial cells and set in a fibrovascular stroma. (B) On higher power, the epithelial cells are focally ciliated. 
Histology of sections taken from the mass attached to the right ovary showed multiple cysts of varying sizes lined by a single layer of epithelial cells (fig 1A). In some areas, the epithelial cells were attenuated, whereas elsewhere they were cuboidal or columnar. On high power examination, many of the cells were ciliated (fig 1B). There was little nuclear pleomorphism and only an occasional mitotic figure was identified, the mitotic count being less than one per 10 high power fields. The cysts were separated by a fibrovascular stroma.

Histology of the two endometrial polyps showed cystically dilated endometrial type glands set in a fibrovascular stroma. The presence of benign leiomyomas was confirmed and there was evidence of adenomyosis. Histology of the ovaries showed each to contain a benign serous cystadenoma.

\section{Discussion}

Clement and Young, in describing four cases of florid cystic endosalpingiosis presenting as a tumour-like mass, drew attention to this condition. ${ }^{2}$ Their cases included two with full thickness involvement of the cervix and lower uterine body, the first reported cases of endosalpingiosis involving the uterus. The authors reviewed the previously reported cases of endosalpingiosis associated with a tumour-like mass on clinical or gross examination and concluded that endosalpingiosis, although usually a microscopic finding, can rarely present as a clinically or grossly evident mass that can be confused with a neoplasm.

The differential diagnosis of cystic endosalpingiosis was discussed in detail by Clement and Young and it is not the purpose of the present report to repeat this. The lesion we describe here is unlikely to be mistaken for malignancy on gross inspection, and the chief diagnostic consideration was an extraovarian serous cystadenoma. ${ }^{3}$ However, the latter is usually unilocular, in contrast to the multicystic nature of the present lesion.

Ordinary endosalpingiosis is particularly common in association with serous tumours of the ovary, and in the present case the woman had bilateral ovarian serous cystadenomas. She had previously been taking tamoxifen for breast cancer. It is well known that tamoxifen may result in a variety of gynaecological complica- tions owing to its weak oestrogenic effects. These include the development of endometrial proliferative lesions (including hyperplasias and adenocarcinomas) and endometrial polyps. ${ }^{4}$ The woman in this case had several endometrial polyps removed previously and there were two polyps in the hysterectomy specimen. There was also adenomyosis, which is relatively rare in postmenopausal women but which may possibly be associated with tamoxifen use. ${ }^{5}$ One of us (WGM) has seen cystic change in adenomyosis in a patient taking tamoxifen; it is tempting to speculate that in the present case the endosalpingiosis underwent proliferation with subsequent cystic transformation in association with the use of this drug. Proliferative changes in endometriosis have been described in patients receiving tamoxifen and have been ascribed to the oestrogenic effects of this. ${ }^{6}$ Interestingly, of the four cases described by Clement and Young, one patient had a history of breast cancer. It is not stated whether she was receiving tamoxifen.

In summary, we describe a case of paraovarian cystic endosalpingiosis which resulted in a grossly visible mass. Pathologists may not be aware of the condition and this may result in diagnostic confusion. The patient had been taking tamoxifen for breast cancer and, although this may be a chance association, it is possible that the drug contributed to the development of cystic change within the endosalpingiosis. As more patients receive tamoxifen, which is now being considered as prophylaxis against breast cancer in high risk women, additional unusual proliferative lesions may be encountered in the female genital tract.

1 Zinsser KR. Endosalpingiosis in the omentum. A study of autopsy and surgical material. Am $\mathcal{F}$ Surg Pathol 1982;6:109-17.

2 Clement PB, Young RH. Florid cystic endosalpingiosis with tumor-like manifestations. A report of four cases including the first reported cases of transmural endosalpingiosis of the first reported cases of transmural endosal
the uterus. Am $\mathcal{7}$ Surg Pathol 1999;23:166-75.

3 de Peralta MN, Delahoussaye PM, Tornos CS, et al. Benign retroperitoneal cysts of müllerian type: a clinicopathologic retroperitoneal cysts of mülerian type: a clinicopathologic
study of three cases and review of the literature. Int $\mathcal{F}$ Gyne-

4 Ismail SM. Pathology of endometrium treated with tamoxifen. F Clin Pathol 1994;47:827-33.

5 Cohen I, Beyth Y, Tepper R, et al. Adenomyosis in postmenopausal breast-cancer patients treated with tamoxifen - a new entity. Gynecol Oncol 1995;58:86-91.

6 Ismail SM, Maulik TG. Tamoxifen-associated postmenopausal endometriosis. Histopathology 1997;30:187-91. 